\title{
SARS-CoV-2 management in emergency department: Risk stratification and care setting identification proposal based on first pandemic wave in Pisa University Hospital
}

\author{
Greta Barbieri, ${ }^{1,2}$ Alessandro Cipriano, ${ }^{2}$ Stella Carrara, ${ }^{3}$ Stefano Spinelli, ${ }^{2}$ Francesco Cinotti, ${ }^{2}$ \\ Francesca Foltran, ${ }^{4}$ Matteo Filippi, ${ }^{4}$ Ferruccio Aquilini, ${ }^{4}$ Michele Tonerini, ${ }^{5}$ Massimo Santini, ${ }^{2}$ \\ Paolo Malacarne, ${ }^{3}$ Lorenzo Ghiadoni ${ }^{2,6}$ \\ ${ }^{1}$ Department of Surgical, Medical, Molecular and Critical Area Pathology, University of Pisa, Pisa; \\ ${ }^{2}$ Emergency Medicine Department; ${ }^{3}$ Department of Anaesthesia and Critical Care Medicine; ${ }^{4}$ Medical \\ Directional Department; ${ }^{5}$ Department of Emergency Radiology, Pisa University Hospital, Pisa; ${ }^{6}$ Department \\ of Clinical and Experimental Medicine, University of Pisa, Italy
}

\begin{abstract}
COVID-19 patients require early treatment and admission to an appropriate care setting, considering possible rapid and unpredictable to Severe Acute Respiratory Syndrome. A flow-chart was developed by a multidisciplinary team of Emergency Department
\end{abstract}

Correspondence: Greta Barbieri, Department of Department of Surgical, Medical, Molecular and Critical Area Pathology, University of Pisa, Via Savi 10, 56126 Pisa, Italy.

Tel.: +39.3470104897

E-mail: greta.barbieri@phd.unipi.it

Key words: COVID-19; ventilation; pneumonia; emergency department; management strategy.

Conflict of interest: The authors declare no conflict of interests. GB and LG are members of ECJ editorial board.

Contributions: GB was responsible for drafting, data analysis, and reviewing the manuscript. PM, LG, and AC designed the study and revised the manuscript. SC was responsible for data collection. SS, FC, FF, MF, FA, MS and MT revised the manuscript.

Availability of data and material: The data are available from the authors upon reasonable request.

Ethics approval and consent to participate: Not applicable.

Informed consent: Not applicable.

Conference presentation: The work was presented as oral communication at Webinar "L'approccio del DEA alla luce delle più recenti Linee Guida" (7 November 2020, Pisa, Italy) and at Webinar "Conseguenze a breve e lungo termine del COVID" (11 Novembre 2021, Pisa, Italy).

Received for publication: 16 May 2019.

Revision received: 29 November 2021.

Accepted for publication: 29 November 2021.

This work is licensed under a Creative Commons Attribution 4.0 License (by-nc 4.0).

COPyright: the Author(s), 2021

Licensee PAGEPress, Italy

Emergency Care Journal 2021; 17:9859

doi:10.4081/ecj.2021.9859
(ED) clinicians, intensivists and radiologists aiming to provide tools for disease severity stratification, appropriate ventilation strategy and hospitalization setting identification. We conducted a retrospective application of our model on 313 hospitalized patients at Pisa University Hospital including 222 patients admitted to ED for respiratory failure between March and April 2020. Risk stratification score was based on respiratory and chest imaging parameters, while management strategy on comorbidities and age. Age, comorbidities, clinical respiratory and arterial blood gas parameters, semi-quantitative chest computed tomography score were significant predictors of mortality $(\mathrm{p}<0.05)$. Mortality rate was higher in patients treated in intensive care units $(26.5 \%)$ and undergoing endo-tracheal intubation $(32.7 \%)$, compared to medical area $(21.3 \%)$. We verified a good concordance $(81.7 \%)$ between the proposed model and actual evaluation in ED. Outcomes analysis of subgroups of patients homogeneous for baseline features allowed to verify safety of our model: in non-elderly and/or non-comorbid patients (15\% mortality) our scheme overestimates the risk in $30 \%$ of cases, but it suggests non-intensive management in patients with reduced functional reserve, elderly and with comorbidities $(50 \%$ mortality). Correct management of respiratory failure COVID-19 patients is crucial in this unexpected pandemic. Our flow-chart, despite retrospectively application in small sample, could represents a valid and safe proposal for evaluation in ED.

\section{Introduction}

\section{Background}

The rapid spread of CO-rona VI-rus D-isease 2019 (COVID19) cases resulted in overcrowd of Emergency Departments (ED). ${ }^{1}$ Even though most patients with COVID-19 infection has minor symptoms and good prognosis, a considerable group shows a Severe Acute Respiratory Syndrome Coronavirus 2 related (SARS-CoV-2) ${ }^{2}$, which requires early treatment and admission to an appropriate care setting, in order to avoid negative outcomes. ${ }^{1,3}$

The official guidelines of Italian Society of Anesthesia Analgesia Resuscitation and Intensive Care (Società Italiana di Anestesia Analgesia Rianimazione e Terapia Intensiva, SIAARTI), published on the $6^{\text {th }}$ of March 2020, drew physician's attention to an epidemic scenario of such magnitude that might cause an imbalance between the population real clinical needs and the effective availability of Intensive Care Unit (ICU) resources. ${ }^{4}$ Considering this possible derangement, the use of appropriate clinical tools is 
determinant to avoid decisions based only on medical resources availability (hospital beds or ventilators).

\section{Importance}

Considering these issues, ED management should provide prompt recognition of unstable or evolutive conditions, given possibility of a rapid and unpredictable clinical deterioration, early identification of the most correct ventilation strategy and wise choice of the most appropriate care setting, balancing limited resources, and preventing ED over-crowding. ${ }^{5}$ From this point of view, the identification of anamnestic, clinical, or instrumental features predicting negative outcomes in COVID-19 patients could represent valuable tools for risk stratification. ${ }^{6-8}$

Despite prognosis of SARS-CoV-2 related disease are not yet completely understood and clinical presentation is variable ranging from asymptomatic carriers to lethal respiratory syndrome, many studies identified age, underlying comorbidities (i.e. chronic cardiovascular and respiratory diseases) as predictors of mortality. ${ }^{3,9}$

The development of respiratory failure is the most fearful complication. ${ }^{10}$ For this reasons, dyspnoea and respiratory parameters are fundamental to predict disease severity. ${ }^{11}$ Therefore, some clinical parameters could reveal respiratory deterioration, which requires ICU admission. ${ }^{6,7}$ Imaging can be helpful to stratify clinical impact of the respiratory syndrome. Chest X-ray has low sensitivity, ${ }^{12}$ while a visual semi-quantitative evaluation or software quantification of disease extent at chest Computed Tomography (CT) shows correlation with clinical severity, ${ }^{13-15}$ and predictors of ICU admission or death. ${ }^{16}$ Moreover, bedside lung ultrasound (LUS) may play a role in COVID-19 pneumonia diagnosis, considering its logistical advantages, in determining the patient's prognosis and in the decision-making process. ${ }^{17-20}$

\section{Goals of investigation}

The aim of our study is to propose a new model of patients' management in ED, providing a risk stratification of COVID-19 patient with respiratory failure, which can suggest ventilation strategy and a care setting based on clinical history, symptoms, respiratory parameters, and imaging. Furthermore, has been verified the applicability, appropriateness, and safety of the proposed model through outcome analysis on population of the first pandemic wave in our hospital.

\section{Material and Methods}

\section{Setting}

The Pisa University Hospital, Italy, is one of the three Regional tertiary-care hospitals, serving as local Primary Health Care center for Pisa (approximately 100.000 inhabitants) and hub center for the North-West part of Tuscany (approximately 1 million 200 thousand inhabitants). The first patient affected by SARS-CoV-2 related respiratory disease was admitted on the $5^{\text {th }}$ of March 2020, 2 weeks later the beginning of SARS-CoV-2 outbreak in the Italian region of Lombardy. ${ }^{21}$

\section{Study design: Proposed flow-chart description}

ED clinicians, intensivists and radiologists of Pisa University Hospital developed a management flow-chart for respiratory failure COVID-19 patients. This model was designed on information collected from other EDs already facing up with the infection outbreak for would trying to summarize poor evidences in literature available on March 2020. The multidisciplinary team "empirical- ly" identified elements to evaluate and stratify the disease severity, according to age, comorbidities, Arterial Blood Gases analysis $(\mathrm{ABG})$, respiratory dynamics and chest imaging.

A final score (PISA CoViD Triage Score - PiCoTS) consists of two consecutive sections: i) disease severity risk stratification, based on respiratory and chest imaging elements; ii) identification of the most appropriate management on ventilation strategy and care setting for the patients, considering their anamnestic characteristics and disease severity.

Section relating to risk stratification (Table 1), provides for assignment of a positive numerical score on 4 items relating to AGB parameters. Two ABG values measured in ambient air and 2 measured after oxygen therapy administration were used for highlighting the ability to correct respiratory failure (Table 1).

Imaging was entered into our model as chest CT data converted to quantitative value using Total Severity Score (TSS), ${ }^{14,15}$ which assigns an increasing numerical value with lung inflammatory involvement, divided by each lobe, allowing multiple levels of severity identification. Each of the five lung lobes was assessed for percentage of lobar involvement and classified as none $(0 \%)$, minimal (1-25\%), mild (26-50\%), moderate (51-75\%), or severe (76$100 \%$ ), corresponding to score of $0,1,2,3,4$. Final TSS score was reached by summing five lobe scores (from 0 to 20). Our risk stratification model assigned a negative score to the category with low lung commitment and an increasing score in the other classes.

Finally, a positive score was assigned to respiratory clinical parameters, respiratory rate $(\mathrm{RR})>25$ acts/minute and-or signs of respiratory distress. The total score obtained stratifies disease in mild (if the score is $<3$ ) or severe (if the score is $\geq 3$ ).

The second step of the flow-chart identifies groups of patients based on their characteristics (age and comorbidities) for application of specific ventilation and hospitalization settings management (Figure 1). Comorbidities (cardiovascular disease, hypertension, diabetes mellitus, respiratory disease, cerebrovascular pathology) were considered significant if present in number $\geq 3$. To provide additional indicators for ventilation strategy choice, we proposed a qualitative and functional assessment of the lung by thoracic imaging (chest TC or LUS bedside) for obtaining information

Table 1. Risk stratification in proposed model. The table identifies severity of disease based on the final score obtained from the sum of the items considered. A score lower than 3 defined a mild severity whereas a score greater than or equal to 3 a severe disease. PaO2: Arterial partial pressure of oxygen; PCO2: Partial pressure of carbon dioxide; P/F: Arterial partial pressure of oxygen / Fractional inspired oxygen ratio; TSS: Total Severity Score; RR: Respiratory rate.

\section{BLOOD GASES}

\begin{tabular}{ll}
$\mathrm{PaO} 2<60 \mathrm{mmHg}$ ADMISSION & +1 \\
$\mathrm{PCO} 2<30 \mathrm{mmHg}$ ADMISSION & +1 \\
$\mathrm{PCO} 2<35 \mathrm{mmHg}$ AFTER O2 THERAPY & +1 \\
$\mathrm{P} / \mathrm{F}>200$ AFTER O2 THERAPY & +1 \\
\hline $\mathrm{CT}$ CHEST IMAGING $\rightarrow$ INIS score &
\end{tabular}

TTS $<5$

\begin{tabular}{lc} 
TTS $5-9$ & +1 \\
\hline TTS $10-14$ & +2 \\
TTS $>15$ & +3 \\
\hline RESPIRATIORY PARAMETERS &
\end{tabular}

$\mathrm{RR} \geq 25$ and/or clinical signs of respiratory distress +1 
about prevailing pattern (interstitial or consolidative), distribution of pneumonia (sub-pleural, ubiquitous) and diaphragmatic function (motility, shortening).

Accordingly, we identified four groups and five categories of patients with a specific indication for management:

A. Severe disease, patients $<70$ years or $70-80$ years WITHOUT comorbidities: i) Category 1: Patients not elderly or without pathologies, indication to endo-tracheal intubation (ETI) in ED and indication to intensive care setting; ii) Category 2: Patients not elderly or without pathologies, indication to Continuous Positive Airway Pressure (CPAP) in ED indication to intensive care setting.A choice between the 2 approaches based on clinical judgment and additional instrumental data is recommended.

B. Mild disease, patients $<70$ years or $70-80$ years WITHOUT comorbidities: i) Category 3: Patients not elderly or without pathologies, indication to oxygen therapy in ED and possible intensive care setting; ii) Category 4: Patients not elderly or without pathologies, indication to oxygen therapy in ED and medical area hospitalization. A choice between the 2 approaches based on clinical judgment after the first hours of treatment and additional instrumental data is recommended.

C. Severe disease, patients $>80$ years or 70-80 years with comorbidities

D. Mild disease, patients $>80$ years or 70-80 years WITH comorbidities.
Both group C and D identify Category 5: Patients elderly or with pathologies, with indication to oxygen therapy as a "maximum of care" provided in ED and admission to medical area.

\section{Application in study population}

Empirical, non-standardized, evaluation based on the above elements was used for management of COVID-19 patients in ED during first pandemic wave. Subsequently, a detailed retrospective statistical analysis of data relating to this group of patients was conducted, with the aim of verifying the applicability, appropriateness, safety, and outcomes of the proposed model.

Clinical data were extracted by ED (FirstAid ${ }^{\circledR}$ ) and hospital-

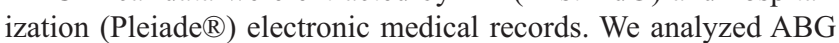
data only in the group of patients with respiratory failure, since in the other subjects there were frequently missing data. Chest CT was available in 269 out of 313 patients (86\%) admitted to ED. Radiologists calculated retrospectively TSS score to allow analyzes on quantitative data. Finally, in 206 patients with full data available, the proposed flow-chart was retrospectively calculated to verify the agreement with the evaluations actually performed in ED.

\section{Analysis}

Continuous variables are expressed, depending on the type of distribution, as mean \pm Standard Deviation (SD) or median and Interquartile Ranges (IQRs). The normality distribution was assessed by the Kolmogorov-Smirnov test. The continuous vari-

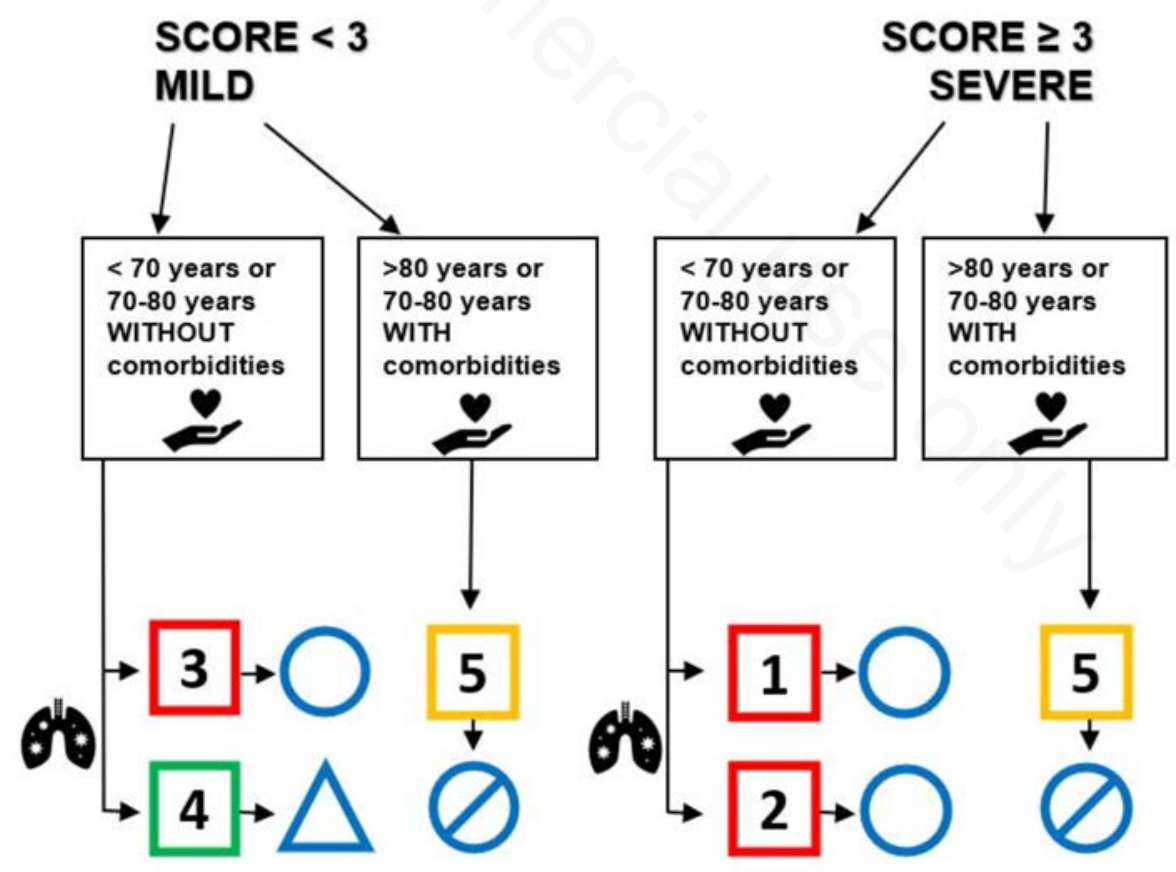

Figure 1. Ventilation and care setting management in proposed model. Heart symbol: Comorbidities. Pathologies are considered significant if present greater than or equal to 3 among diabetes, respiratory pathology, cardio and cerebro-vascular disease. Lung symbol: Qualitative and functional evaluation of thoracic imaging, concerning pneumonia pattern (interstitial, consolidative), distribution and diaphragm, to be considered for the choice of the ventilation strategy. Square: ventilation strategy. Red: ETI or CPAP; green: oxygen therapy; yellow: ETI not indicated. Circle/triangle: setting. Open circle: useful/possible intensive care setting; tringle: medical area; crossed out circle: intensive care setting not indicated. Category 1: Patients not elderly or without pathologies, indication to ETI in ED, useful or possible intensive care setting. Category 2: Patients not elderly or without pathologies, indication to CPAP in ED, indication to intensive care setting. Category 3: Patients not elderly or without pathologies, indication to oxygen therapy in ED, possible intensive care setting. Category 4: Patients not elderly or without pathologies, indication to oxygen therapy in ED and medical area hospitalization. Category 5: Patients elderly or with pathologies, indication to oxygen therapy in ED, intensive care setting not indicated. 
ables were evaluated with the Mann - Whitney test. The categorical variables are expressed across frequency distributions and chisquare was used to establish the difference between groups. Statistical significance was set for $\mathrm{p}<0.05$. All the results obtained were analyzed with statistical software (NCSS).

\section{Patient and public involvement}

As we conducted a retrospective study during a pandemic period, patient and public involvement was not possible.

\section{Results}

\section{Characteristics of study subjects}

In the period from March $5^{\text {th }}$ to April $30^{\text {th }} 2020$, a total of 313 COVID-19 patients were hospitalized in University Hospital of Pisa. Of these, 222 were evaluated in ED with the aid of intensivist consulting, due to clinical presentation with respiratory distress.

Gender and demographics characteristics showed a prevalence of males among patients hospitalized for COVID-19 (209 out of $313,66.8 \%$ ). Mean age was 67 years, with men younger than women (66 versus 70 years). Patients with respiratory failure at ED admission showed mean age of 68 years, 66 years in the 144 men $(65 \%)$ and 70 years in the 78 women, respectively.

\section{Prediction of flow-chart items}

We analysed each item present in our risk stratification scheme dividing the population between survived and deceased patients, in order to verify their ability to predict the negative outcome. An alteration of clinical and $\mathrm{ABG}$ parameters were more frequently present ( $\mathrm{p}$ value $<0.05$ ) in patients with adverse outcome (Table 2). As regards chest imaging, we observed that TSS value, was significantly higher in deceased patients (average values $12.09 \pm 4.92$ ) than in the survivors (average values $8.86 \pm 4.44$ ) in the group of patients with respiratory failure. This difference was also observed in total population of hospitalized COVID-19 patients (average values in deceased patients was $11.6 \pm 5.6$ and in discharged patients was 8.2 \pm 4.9 ), as shown in Figure 2 .

Comorbidities (cardiovascular and cerebrovascular disease, hypertension, diabetes mellitus, respiratory disease) were significantly associated to mortality in COVID-19 patients assessed in ED for respiratory failure and those hospitalized globally (Table 3 ). In addition, mean age is significantly higher in deceased patients, in both groups (Table 3).

Dividing COVID-19 respiratory failure population according to our risk stratification score with cut-off of 3 , we observed a positive outcome in $63 \%$ of cases of patients with score $<3$, compared to the group with a score $\geq 3$, in which survival was $37 \%$. This difference is statistically significant $(\mathrm{p}>0.05)$

Table 2. Outcome Predictors in COVID-19 patients with Respiratory Failure. Blood gas analysis and respiratory parameters in the population according to mortality. PaO2: Arterial partial pressure of oxygen; PCO2: Partial pressure of carbon dioxide; P/F: Arterial partial pressure of oxygen / Fractional inspired oxygen ratio; TSS: Total Severity Score; RR: Respiratory rate.

\begin{tabular}{lccc}
\hline Outcome Predictors in COVID-19 patients with Respiratory Failure & $\begin{array}{c}\text { Discharged } \\
\text { N./lot. (\%) }\end{array}$ & $\begin{array}{c}\text { Deaths } \\
\text { N./lot. (\%) }\end{array}$ & p value \\
$\mathrm{PaO}<60 \mathrm{mmHg}$ at presentation & $51 / 135(37.8)$ & $22 / 30(73.3)$ & $<0.001$ \\
$\mathrm{PCO}<30 \mathrm{mmHg}$ at presentation & $33 / 133(24.8)$ & $14 / 29(48.3)$ & 0.012 \\
\hline $\mathrm{PCO}<35 \mathrm{mmHg}$ after oxygen therapy & $37 / 99(37.4)$ & $17 / 25(68.0)$ & 0.006 \\
$\mathrm{P} / \mathrm{F}<200 \mathrm{mmHg}$ after oxygen therapy & $27 / 99(27.3)$ & $21 / 25(84.0)$ & $<0.001$ \\
\hline RR $\geq 25$ breaths/min and/or respiratory distress & $16 / 44(36.4)$ & $12 / 17(70.6)$ & 0.016 \\
\hline
\end{tabular}
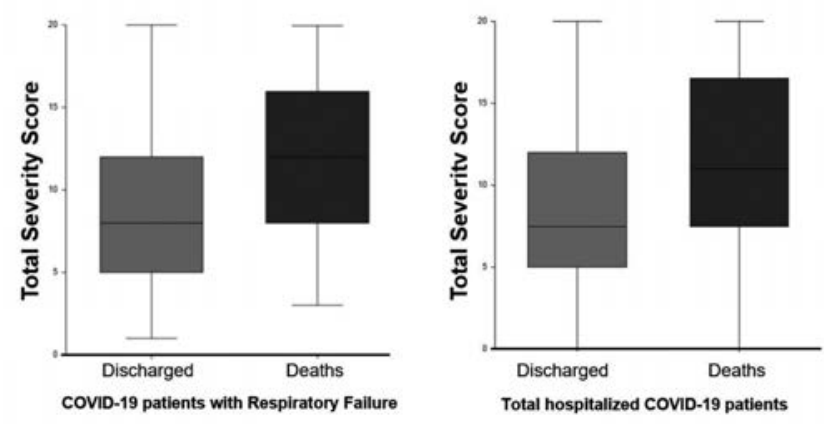

Figure 2. Average total severity score in COVID 19 patients. Respiratory failure patients (left panel) and total of hospitalized (right panel), population divided according to mortality (discharged or death).

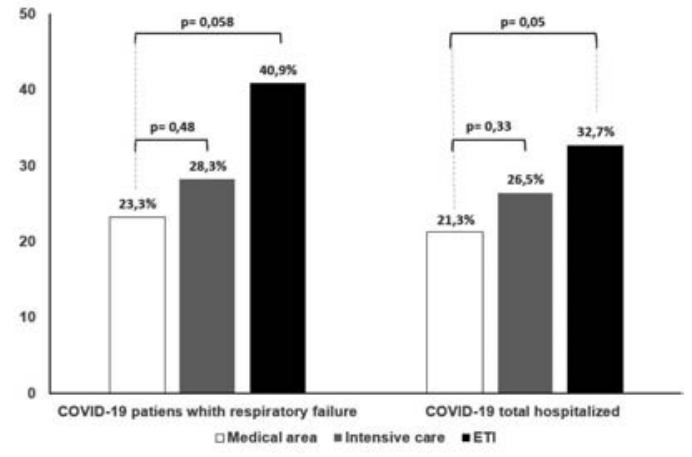

Figure 3. COVID-19 patients' mortality by care setting. Graphs show results on mortality in patients hospitalized in medical area, ICU and ICU undergoing ETI. ETI: Endo-tracheal Intubation. 


\section{Proposed model and real evaluation concordance}

We were able to retrospectively apply our model in 142 patients (who had all the required items of PiCoTS flow-chart). The agreement of the proposed "theoretic" score with the evaluation about patients severity and management actually performed in ED was to $81.7 \%$ (116 out of 142 patients).

\section{Mortality analysis by care setting}

In our population, 230 out of 313 patients $(73.5 \%)$ were hospitalized exclusively in medical area, while 83 were managed in intensive area. Of these 83 patients, 52 (62.7\%) underwent ETI, whereas $31(37.3 \%)$ received exclusively non-invasive ventilation.

When mortality was analysed in various care settings, it was higher in patients managed in an intensive care setting in patients evaluated in the ED for respiratory failure, and in the total of COVID-19 hospitalized patients. The highest mortality was in patients undergoing ETI (Figure 3).

\section{Subcategories analysis}

Patients with low severity score, under the age of 70 or between 70 and 80 years of age and without comorbidities $(n=87$; indication to oxygen therapy as an initial strategy, but possibly eligible for an intensive care setting) showed the lowest mortality rate $(n=6 ; 6.7 \%)$.

Patients with severe disease, under the age of 70 or between 70 and 80 years of age but without comorbidity, $(n=52$; indication for CPAP or ETI in ED, management in intensive care setting) showed a mortality of $15 \%(n=8)$. Among these, a discrepancy between the indication of the proposed score and the evaluation actually performed was demonstrated in 16 out of 52 patients (30.8\%). In each patient, the proposed model overestimated risk. Approximately $40 \%$ of patients $(n=22)$ in this group received an indication to CPAP in ED, which was not performed in ED, but subsequently during hospitalisation, in intensive care setting.

Finally, the analysis of COVID- 19 patients over 80 or $70-80$ years of age with comorbidity ( $n=67$; indication of oxygen therapy as maximum intensity of care, not eligible for management in intensive care setting) shows a mortality of $50 \%(n=34)$. In $93 \%$ of cases, this group of patients was managed in a medical setting. However, even the small group of patients to whom intensive care was available, 4 out $5(80 \%)$ had a negative outcome.

\section{Discussion}

During the COVID-19 pandemic, ED clinicians have to cope with the decision of offering the more appropriate intensity and thereafter setting of care, sometimes in situation of reduced healthcare resource. The management of a potentially rapidly worsening condition demands even more an effective triage systems and care's pathways. Identifying and treating appropriately patients with rapidly evolving disease is crucial in this unexpected pandemic. ${ }^{5,7}$ A large scientific literature has emerged in recent months for identification of negative prognostic factors in patients with COVID-19 pneumonia, based on comorbidities, clinical and respiratory parameters. ${ }^{3,6,7}$ The present analysis also identified as predictors of death within our population, ABG parameters (suggestive for hypoxia and inadequate response to oxygen therapy), clinic Respiratory Parameters (RR), age and pre-existing pathologies (chronic respiratory, cardio/cerebrovascular disease, diabetes, hypertension), visual semi-quantitative evaluation of disease extent at chest $\mathrm{CT}$, in accordance with previous reports from the literature. ${ }^{7,14-16,22-24}$

The proposed flow-chart, providing the use of these indicators for stratifying patient's risk during first ED evaluations, aimed to identify non-arbitrary elements to be verified subsequently in our population. Our approach is in line with other studies that suggest stratifying the severity of disease based on comparable respiratory parameters. . $^{6,22}$

A multidisciplinary teamwork allowed to "outline" our model and retrospectively tested it on data of the first pandemic wave in University Hospital of Pisa. We verified the concordance between the evaluations actually applied during the first pandemic wave and those "theoretical" and standardized applied by the retrospective application of the flow-chart.

The overall mortality in our sample $(22.7 \%)$ and the mortality of patients hospitalized in ICU $(26.5 \%)$ were in line to the mortality reported in the literature, with a comparable age and gender distribution of other Italian hospitals. We observed a substantial difference between the mortality rates of patients managed in a low intensity and those managed in an intensive care setting. This gap could be explained by a greater severity of disease presentation in the group underwent intensive care management. However, higher

Table 3. Outcome Predictors in COVID-19 patients with Respiratory Failure and total hospitalized patients.

\begin{tabular}{|c|c|c|c|}
\hline Outcome Predictors & Discharged & Deaths & p value \\
\hline COVID-19 patients with Respiratory Failure (N. 222) & N. $168(\%)$ & N. 540 & \\
\hline Cardiovascular disease & $12(7.1)$ & $23(42.6)$ & $<0.01$ \\
\hline Hypertension & $31(18.5)$ & $22(40.7)$ & $<0.01$ \\
\hline Diabetes mellitus & $14(8.3)$ & $13(24.1)$ & $<0.01$ \\
\hline Chronic lung disease & $11(6.5)$ & $9(16.7)$ & 0.024 \\
\hline Cerebrovascular disease & $6(3.6)$ & $6(11.1)$ & 0.033 \\
\hline Age & $64 \pm 16$ & $80 \pm 11$ & $<0.01$ \\
\hline Total hospitalized COVID-19 patients (N. 313) & N. 242() & N. 710 & \\
\hline Cardiovascular disease & $68(28.1)$ & $37(52,1)$ & $<0.01$ \\
\hline Hypertension & $99(40,9)$ & $44(62)$ & $<0.01$ \\
\hline Diabetes mellitus & $44(18.2)$ & $60(19.2)$ & $<0.01$ \\
\hline Chronic lung disease & $17(7)$ & $20(28,2)$ & $<0.01$ \\
\hline Cerebrovascular disease & $22(9)$ & $17(23.9)$ & $<0.01$ \\
\hline Age & $64.3 \pm 15.5$ & $78.7 \pm 11$ & $<0.01$ \\
\hline
\end{tabular}


mortality of subjects subjected to ETI raises the question about appropriateness of choosing this ventilation strategy in the face of non-invasive approaches. Indeed, ventilation management of COVID-19 patients with respiratory failure is still controversial and no clear scientific evidence is currently available. Gattinoni et al. described lung damage in the affected patient from COVID-19 pneumonia similar in some respects to Acute Respiratory Distress Syndrome (ARDS). ${ }^{25}$ They were recognized in this context two different pulmonary pictures on the basis of respiratory compliance, which suggest different management in terms of ventilatory strategy. ${ }^{25,26}$ Furthermore, our model, based on anamnestic, demographic baseline characteristics with additional lung and diaphragmatic imaging tools ${ }^{18}$ useful for focusing the disease phase, suggests a possible appropriate ventilatory strategy and hospitalization setting, in line with literature ${ }^{17,27}$ and resuscitation recommendations. ${ }^{4}$

The analysis of patients with low severity score, under the age of 70 or between 70 and 80 years of age without comorbidity, shows the lowest mortality rate $(6.7 \%)$, despite a proportion of these patients needed intensive treatments. Auxiliary evaluation in the view of a possible increase in intensity of care in this group can be represented by LUS, by identifying of specific patterns (consolidative or interstitial) ${ }^{18}$ and evaluating of diaphragmatic function. ${ }^{27}$

The analysis of patients with high severity score, under the age of 70 or between 70 and 80 years of age without comorbidity shows a high mortality rate $(15 \%)$, related to the severity of disease in the younger group and/or the relatively good clinical baseline conditions. It is to be highlighted that a discrepancy between the indication of proposed score and the evaluation performed during first pandemic wave was observed in some patients. Indeed, the proposed model overestimates patient's risk, suggesting that an intensive management could be implement in this subgroup of healthy and non-elderly patients and also a good "safety" of the model. Regarding ventilation treatments, approximately $40 \%$ of patients in this group had received an indication to CPAP in ED, which has not applied for organizational problems in our setting. ${ }^{21}$ However, it was applied later on, during hospitalization. These considerations provide useful tips for better management during the new pandemic wave, including LUS, which can be used not only for diagnostic purposes, but also to guide ventilation choice (through evaluation of prevailing patterns and diaphragmatic function). ${ }^{17,18,27}$

Finally, the analysis of COVID-19 patients over 80 or $70-80$ years of age with comorbidity, shows a high mortality rate (approximately 50\%), which is not different when patients undergo ICU. This suggests that a low care is more appropriate for this group, in accordance with ethical considerations and available recommendations. ${ }^{4}$ A strong functional reserve is necessary to overcame long and aggressive ICU treatment, which is why it is useful to delineate a "limit" to care in these subjects, as our model suggests. Indeed, patients of this group should be managed in the medical area, since intensive care treatments did not improve outcomes in our populations. Therefore, a non-intensive setting could represent a valid alternative, in which CPAP can be applied in selected cases as a possible therapeutic attempt.

\section{Limitations and perspectives}

Among the limitations of this study, the small sample size should be considered. The proposed methodology was outlined empirically by experts in the sector (due to the lack of scientific references) and only subsequently verified through statistical analyzes. In addition, the proposed methodology was verified on population of a single center and retrospectively. This may have result- ed in observation of negative effects related to organizational problems of our hospital, not necessarily related to ED evaluation phase.

A possible application of our flow-chart could be the prospective use in patients of second pandemic wave in our ED and in other geographical areas, with the aim of comparing the outcomes between the "empirical" and the "standardized" approach.

\section{Conclusions}

A tool which integrates the physiological patterns of disease, by clinical and $\mathrm{ABG}$ parameters, imaging findings, together with patient's underlying conditions, may prove useful to ED physicians, to stratify COVID-19 patient's risk early and to choose the appropriate level of care and ventilation strategy. Our model, despite the limitations of low number and retrospective application, meets to these needs through a systematic and multidisciplinary evaluation.

\section{References}

1. WHO. Clinical management of COVID-19: interim guidance. WHO: 27 May 2020. 2020. Available from: https://apps.who.int/iris/handle/10665/332196

2. Guan WJ, Ni ZY, Hu Y, et al. Clinical characteristics of coronavirus disease 2019 in China. N Engl J Med 2020;382:17081720 .

3. Figliozzi S, Masci PG, Ahmadi N, et al. Predictors of adverse prognosis in COVID-19: A systematic review and meta-analysis. Eur J Clin Invest 2020;50:e13362.

4. Gruppo di Studio ad Hoc della Commissione di Bioetica della SIAARTI. SIAARTI guidelines for admission to and discharge from Intensive Care Units and for limitation of treatment in intensive care. Minerva Anestesiol 2003;69:101-11, 111-18.

5. Di Domenico SL, Coen D, Bergamaschi M, et al. Clinical characteristics and respiratory support of 310 COVID-19 patients, diagnosed at the emergency room: a single-center retrospective study. Intern Emerg Med 2020;16:1051-60.

6. Liang W, Liang H, Ou L, et al. Development and validation of a clinical risk score to predict the occurrence of critical illness in hospitalized patients with COVID-19. JAMA Intern Med 2020;180:1081-9.

7. Haimovich AD, Ravindra NG, Stoytchev S, et al. Development and validation of the quick COVID-19 severity index: A prognostic tool for early clinical decompensation. Ann Emerg Med 2020;76:442-53.

8. Swiss Academy Of Medical Sciences. COVID-19 pandemic: triage for intensive-care treatment under resource scarcity (3rd, updated version). Swiss Med Wkly 2020;150:w20401.

9. Zheng Z, Peng F, Xu B, et al. Risk factors of critical \& mortal COVID-19 cases: A systematic literature review and metaanalysis. J Infect 2020;81:e16-e25.

10. Wiersinga WJ, Rhodes A, Cheng AC, et al. Pathophysiology, transmission, diagnosis, and treatment of coronavirus disease 2019 (COVID-19): A review. JAMA 2020;324:782-93.

11. Wu Z, McGoogan JM. Characteristics of and important lessons from the coronavirus disease 2019 (COVID-19) outbreak in China: Summary of a report of 72314 cases from the Chinese Center for Disease Control and Prevention. JAMA 2020;323:1239-42.

12. Cozzi D, Albanesi M, Cavigli E, et al. Chest X-ray in new 
Coronavirus Disease 2019 (COVID-19) infection: Findings and correlation with clinical outcome. Radiol Med 2020;125:730-7.

13. Francone M, Iafrate F, Masci GM, et al. Chest CT score in COVID-19 patients: Correlation with disease severity and short-term prognosis. Eur Radiol 2020;30:6808-6817.

14. Li K, Fang Y, Li W, et al. CT image visual quantitative evaluation and clinical classification of coronavirus disease (COVID-19). Eur Radiol 2020;30:4407-16.

15. Chung M, Bernheim A, Mei X, et al. CT imaging features of 2019 novel coronavirus (2019-nCoV). Radiology 2020;295:202-7.

16. Colombi D, Villani GD, Maffi G, et al. Qualitative and quantitative chest CT parameters as predictors of specific mortality in COVID-19 patients. Emerg Radiol 2020; doi:10.1007/s10140-020-01867-1

17. Gargani L, Soliman-Aboumarie H, Volpicelli G, et al. Why, when, and how to use lung ultrasound during the COVID-19 pandemic: Enthusiasm and caution. Eur Heart J Cardiovasc Imaging 2020;21:941-8.

18. Volpicelli G, Gargani L. Sonographic signs and patterns of COVID-19 pneumonia. Ultrasound J 2020;12:22.

19. Poggiali E, Bastoni D, Ferrari M, et al. COVID-19 or not COVID-19, that is the question. The role of screening criteria and point-of-care lung ultrasound in the triage decision-making process in an Emergency Department during the phase 2 of the COVID-19 Italian epidemic. Emerg Care J 2021;17:9708.

20. Dacrema A, Silva M, Rovero L, et al. A simple lung ultrasound protocol for the screening of COVID-19 pneumonia in the emergency department. Intern Emerg Med 2021;16:1297-305.

21. Barbieri G, Spinelli S, Filippi M, et al. COVID-19 pandemic management at the Emergency Department: The changing scenario at the University Hospital of Pisa. Emerg Care J 2020;16:9146.

22. Knight SR, Ho A, Pius R, et al. Risk stratification of patients admitted to hospital with covid-19 using the ISARIC WHO Clinical Characterisation Protocol: Development and validation of the 4C Mortality Score. BMJ 2020;370:m3339.

23. Di Castelnuovo A, Bonaccio M, Costanzo S, et al. Common cardiovascular risk factors and in-hospital mortality in 3,894 patients with COVID-19: Survival analysis and machine learning-based findings from the multicentre Italian CORIST Study. Nutr Metab Cardiovasc Dis 2020;30:1899-913.

24. Falcone M, Tiseo G, Barbieri G, et al. Role of low-molecular weight heparin in hospitalized patients with SARS-CoV-2 pneumonia: a prospective observational study. Open Forum Infectious Diseases 2020;7:ofaa563.

25. Gattinoni L, Chiumello D, Caironi P, et al. COVID-19 pneumonia: different respiratory treatments for different phenotypes? Intensive Care Med 2020;46:1099-102.

26. Marini JJ, Gattinoni L. Management of COVID-19 respiratory distress. JAMA 2020;323:2329-30.

27. Corradi F, Vetrugno L, Orso D, et al. Diaphragmatic thickening fraction as a potential predictor of response to continuous positive airway pressure ventilation in Covid-19 pneumonia: A single-center pilot study. Respir Physiol Neurobiol 2020;284:103585. 